\title{
CLASIFICACION Y ENMARCACION DEL CONOCIMIENTO EDUCATIVO*
}

\author{
Basil Bernstein
}

\section{Introducción}

Cómo una sociedad selecciona, clasifica, distribuye, transmite y evalúa el conocimiento educativo, que ella considera debe ser público, refleja tanto la distribución del poder como los principios de control social. Desde este punto de vista, las diferencias dentro de, y el cambio en, la organización, transmisión y evaluación del conocimiento educativo debiera ser una importante área de interés sociológico. (Bemstein, B., 1966, 1967; Davies, D.I., 1970a, 1970b; Musgrove, 1968, Hoyle, 1969; Young, M., 1970). Tal estudio, por supuesto, forma parte del amplio problema de la estructura y cambios en la estructura de la transmisión cultural. Por muchas razones, los sociólogos británicos han evitado el estudio de este problema. Como resultado, la sociología de la educación ha sido reducida a una serie de problemas de entrada-salida; la escuela ha sido transformada en una organización compleja, o en una institución procesadora de personas; el estudio de la socialización ha sido trivializado.

El conocimiento educativo es uno de los mayores reguladores de la estructura de la experiencia. Desde este punto de vista, uno puede preguntarse cómo formas de experiencia, identidad y relación social se evocan, se mantienen y cambian mediante la transmisión formal del conocimiento educativo y sus particularidades. El conocimiento educativo formal, se considera, puede ser realizado a través de tres sistemas de mensajes: el curriculum, la pedagogía y la evaluación. El curriculum define lo que cuenta como conocimiento válido, la pedagogía define lo que cuenta como transmisión válida del conocimiento, y la evaluación define lo que cuenta como realización (manifestación) válida de este conocimiento por parte del aprendiz. El término "código del conocimiento educativo" que será introducido más tarde, se refiere a los principios subyacentes que configuran el curriculum, la pedagogía y la evaluación. En este artículo se argumentará que la forma que este código toma depende de principios sociales que regulan la clasificación y la enmarcación del conocimiento que se hace público en las instituciones educativas. Tanto Durkheim como Marx nos han mostrado que la estructura de las clasificaciones y enmarcaciones de la sociedad revela tanto la distribución del poder como los principios de control. Esperamos demostrar, teóricamente, que los códigos educativos proporcionan excelentes oportunidades para el estudio de la clasificación y de la enmarcación a través de los cuales se da una forma distintiva a la experiencia. Este artículo se organiza como sigue:

1. Distinguiremos primero entre dos tipos de currícula: agregado e integrado.

2. Haremos una elaboración teórica a partir de esta distinción con el propósito de establecer un conjunto de conceptos más general.

3. Derivaremos luego una tipología de códigos educativos.

4. Se estudiarán los aspectos sociológicos de dos códigos educativos muy diferentes.

\footnotetext{
* Tomado de: Class, Codes and control, Vol. 1 Towards a Theory of Educational Transmissions London: Routledge and Kegan Paul. 1974. y traducido con permiso del autor por Mario Díaz. 
5. Esto conducirá a la discusión de la relación entre los códigos educativos y los problemas de control social.

6. Finalmente, habrá una discusión de las razones para el debilitamiento de un código y el fortalecimiento del movimiento de otro.

\section{Dos tipos de currícula}

Inicialmente vamos a hablar acerca del curriculum en una forma muy general. En todas las instituciones educativas existe una distribución formal del tiempo en períodos. Estos pueden variar desde 10 minutos hasta tres horas o más. Llamaremos a tal período formal de tiempo una "unidad". Usaremos la palabra "contenido" para describir cómo se usa un período de tiempo. Definiremos curriculum inicialmente en términos del principio por el cual ciertas unidades de tiempo y sus contenidos se presentan en relaciones especiales entre sí. Miraremos ahora más detenidamente la frase "relaciones especiales".

Primero, nosotros podemos examinar las relaciones entre los contenidos en términos de la cantidad de tiempo acordado a un contenido dado. Inmediatamente, podemos ver que a algunos contenidos se dedica más tiempo que a otros. Segundo, algunos contenidos pueden, desde el punto de vista de los alumnos, ser obligatorios u opcionales. Podemos ahora evaluar el estatus relativo de un contenido en términos del número de unidades de tiempo asignadas a éste, y en términos de si éste es obligatorio u opcional. Esto plantea inmediatamente el problema del estatus relativo de un contenido y de su significado en una carrera educativa dada.

Podemos, sin embargo, considerar las relaciones entre contenidos desde otra perspectiva quizás más importante. Podemos preguntamos, en relación con un contenido dado, silos límites entre éste y otro contenido están claramente marcados o borrados. Hasta qué punto los diversos contenidos están suficientemente aislados entre sí. Si los diversos contenidos están claramente aislados los unos de los otros podemos decir que éstos se presentan en una relación cerrada entre sí. Si el aislamiento entre los contenidos es reducido diremos que éstos se presentan en una relación abierta entre sí. Hasta aquí, estamos sugiriendo que podemos penetrar en cualquier institución educativa y examinar la organización del tiempo en términos del estatus relativo de los contenidos, y en términos de silos contenidos se presentan en una relación abierta o cerrada entre sí. Estamos deliberadamente usando este lenguaje muy abstracto con el propósito de enfatizar que no existe nada intrínseco al estatus relativo de los diversos contenidos, ni nada intrínseco a las relaciones entre los contenidos. Independientemente de la lógica intrínseca de las diversas formas de pensamiento público, las formas de su transmisión, esto es. su clasificación y enmarcación, son hechos sociales. Existe un número de medios alternativos de acceso a las formas públicas de pensamiento, y de esta manera a las diversas realidades que ellas hacen posible. Estamos, por lo tanto, enfatizando la naturaleza social del sistema de alternativas de las cuales surge una constelación llamada curriculum. Desde este punto de vista, cualquier curriculum vincula un principio o principios por medio de los cuales entre todos los contenidos de tiempo posibles, a algunos contenidos se les asigna un esta-tus diferencial y se presentan en una relación abierta o cerrada con otros contenidos.

Distinguiremos ahora entre dos grandes tipos de curriculum. Si los contenidos se presentan entre sí en una relación cerrada, esto es, silos contenidos están claramente delimitados y aislados entre sí, llamaremos a tal curriculum curriculum de tipo agregado. Aquí, el aprendiz tiene que coleccionar un grupo de contenidos favorecidos con el 
propósito de satisfacer algunos criterios de evaluación. Puede existir por supuesto algún concepto subyacente a un curriculum de tipo agregado, como por ejemplo, el de caballero, el de hombre educado, el de hombre diestro, el de hombre no vocacional.

Ahora, opondremos al curriculum tipo agregado, un curriculum donde los diversos contenidos no se presentan separados, sino que se presentan en una relación abierta entre sí. Llamaremos a tal curriculum un curriculum de tipo integrado. De esta manera, podemos tener diversos tipos de curriculum agregado y diversos grados y tipos de integración.

\section{Clasificación y enmarcación}

Introduciremos ahora los conceptos clasificación y enmarcación que serán usados para analizar la estructura subyacente de los tres sistemas de mensajes, curriculum, pedagogía y evaluación, los cuales son realizaciones del código del conocimiento educativo. La idea básica está incorporada en el principio usado para distinguir los dos tipos de curricula: agregado e integrado. El fuerte aislamiento entre los contenidos apunta hacia el curriculum tipo agregado, mientras que el aislamiento reducido apunta a un curriculum tipo integrado. El principio regulador en este caso es la fuerza de la delimitación entre los contenidos. La noción de fuerza de delimitación subyace a los conceptos de clasificación y formación ${ }^{20}$.

La clasificación, en este caso, no se refiere a lo que es clasificado sino a las relaciones entre los contenidos. Clasificación se refiere a la naturaleza de la diferenciación entre los contenidos. Cuando la clasificación es fuerte los contenidos están claramente aislados los unos de los otros por fuertes límites. Cuando la clasificación es débil, existe un aislamiento reducido entre los contenidos, pues las fronteras entre éstos son débiles o están borradas. Clasificación, se refiere al grado de mantenimiento de los limites entre contenidos. La clasificación enfoca nuestra atención hacia la fuerza de los límites como el rasgo distintivo crítico de la división del trabajo del conocimiento educativo. La clasificación nos da, como esperamos demostrarlo, la estructura básica del sistema de mensajes denominado curriculum.

El concepto enmarcación se usa para determinar la estructura del sistema de mensajes denominado pedagogía. Enmarcación se refiere a la forma del contexto en el cual el conocimiento se transmite y recibe. Enmarcación se refiere a la relación pedagógica específica, maestro-alumno. En la misma forma en que clasificación no se refiere a los contenidos, enmarcación no se refiere a los contenidos de la pedagogía. Enmarcación se refiere a la fuerza de los límites entre lo que puede ser transmitido y lo que no puede ser transmitido, en la relación pedagógica. Cuando la enmarcación es fuerte hay un límite agudo, cuando la enmarcación es débil el límite entre lo que puede y no puede ser transmitido se borra.

Enmarcación se refiere al rango de las opciones disponibles al maestro y al alumno en el control de lo que se transmite y recibe en el contexto de la relación pedagógica. La enmarcación fuerte articula opciones reducidas; la enmarcación débil articula un amplio número de opciones. De esta manera enmarcación se refiere al grado de control que el maestro y el alumno poseen sobre la selección, organización, ritmo y tiempo del conocimiento que se transmite y recibe en la relación pedagógica.

\footnotetext{
${ }^{20}$ De esto se deduce que la fuerza del emnarcamiento para el maestro y el alumno puede ser evaluada en los diferentes niveles de selección, organización, ritmo y temporalización del conocimiento.
} 
Hay otro aspecto de la relación de delimitación entre lo que puede ser enseñado y lo que no puede ser enseñado, y por consiguiente, otro aspecto de la enmarcación. Podemos considerar la relación entre el conocimiento cotidiano del maestro y del alumno y el conocimiento transmitido en la relación pedagógica. Podemos plantear la pregunta sobre la fuerza de la delimitación o el grado de aislamiento entre el conocimiento cotidiano comunitario del maestro y del alumno y el conocimiento educativo. De esta manera, podemos considerar las variaciones en la fuerza de la enmarcación con referencia a la fuerza de la delimitación entre el conocimiento educativo y el conocimiento cotidiano comunitario del maestro y del alumno.

Desde la perspectiva de nuestro análisis, la estructura básica del sistema de mensajes denominado curriculum es dada por las variaciones en la fuerza de la clasificación y la estructura básica del sistema de mensajes denominado pedagogía es dada por las variaciones en la fuerza de la enmarcación. Más adelante se demostrará que la estructura del sistema de mensajes denominado evaluación es una función de la fuerza de la clasificación y de la enmarcación. Es importante aclarar que la fuerza de la clasificación y la fuerza de la enmarcación pueden variar independientemente la una de la otra. Así, por ejemplo, es posible tener una clasificación débil con una enmarcación excepcionalmente fuerte. Considérese la instrucción programada. Aquí, la delimitación entre los contenidos educativos puede estar borrada (clasificación débil) pero hay muy poco control por parte del alumno (excepto en el ritmo) sobre lo que se aprende (enmarcación fuerte). Este ejemplo también muestra que la enmarcación puede ser examinada en diferentes niveles y que la fuerza puede variar entre los diferentes niveles de selección, organización, ritmo y temporalización del conocimiento transmitido en la relación pedagógica.

Nos gustaría también aclarar el componente "poder" de este análisis (éste será desarrollado de manera más completa más adelante) y lo que podría llamarse el componente "identidad". Cuando la clasificación es fuerte las fronteras entre los diferentes contenidos están fuertemente marcadas. Cuando éste es el caso, esto presupone la existencia de mantenedores de la delimitación fuerte. La clasificación fuerte también crea un fuerte sentido de pertenencia a una clase particular y de esta manera constituye una identidad específica. La enmarcación fuerte reduce el poder del alumno sobre el "qué", el "cuándo", el "cómo", del conocimiento que él recibe e incrementa el poder del maestro en la relación pedagógica. Sin embargo, la clasificación fuerte reduce el poder del maestro sobre lo que transmite ya que éste no puede sobrepasar los límites entre los contenidos La clasificación fuerte también reduce el poder del maestro en comparación con el poder de los mantenedores de los límites.

Ahora es posible hacer explícito el concepto de código del conocimiento educativo. El código se define en el nivel más general por las relaciones entre la clasificación y la enmarcación.

\section{Una tipología de los códigos del conocimiento educativo}

A la luz del marco conceptual que hemos desarrollado, usaremos la distinción entre curriculum agregado y curriculum integrado con el propósito de elaborar una tipología de los tipos y sub-tipos de códigos educativos La base formal de la tipología se constituye por la fuerza de la clasificación y de la enmarcación. Sin embargo, los subtipos serán distinguidos, inicialmente, en términos de diferencias substantivas (sustanciales).

Cualquier organización del conocimiento educativo que envuelva una clasificación fuerte da origen a lo que aquí se llama un código agregado. Cualquier organización del 
conocimiento educativo que envuelva un marcado intento para reducir la fuerza de la clasificación aquí se llama código integrado. Los códigos agregados pueden dar origen a una serie de subtipos, cada uno de los cuales varían en la fuerza relativa de la clasificación y de la enmarcación. Los códigos integrados también pueden variar en términos de la fuerza de la enmarcación con referencia al control que el maestro o los alumnos pueden tener del conocimiento que se transmite.

La siguiente figura presenta los rasgos generales de la tipología.

Tipo

Modo

$\underline{\text { Variedad }}$

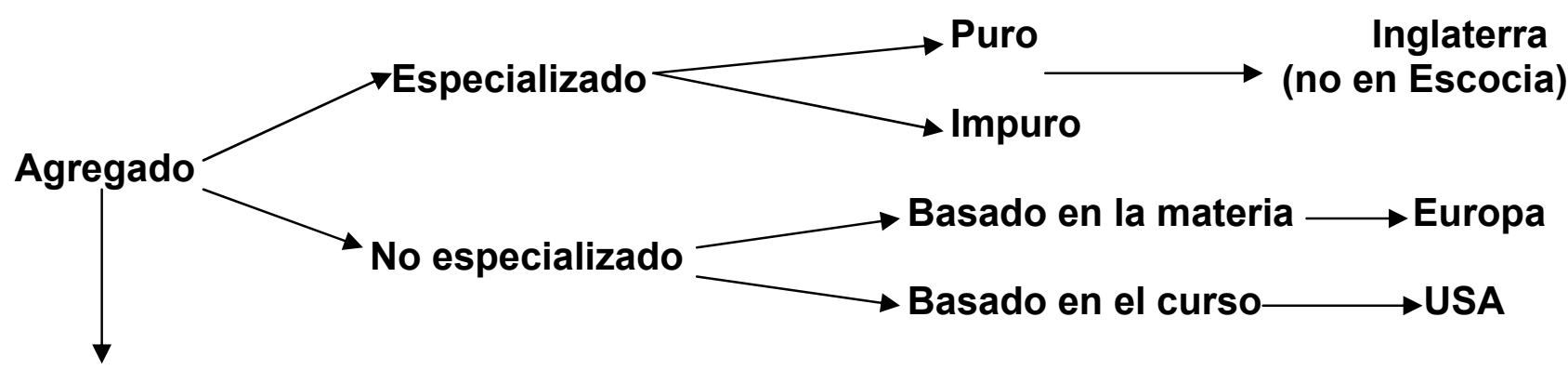

De clasificación

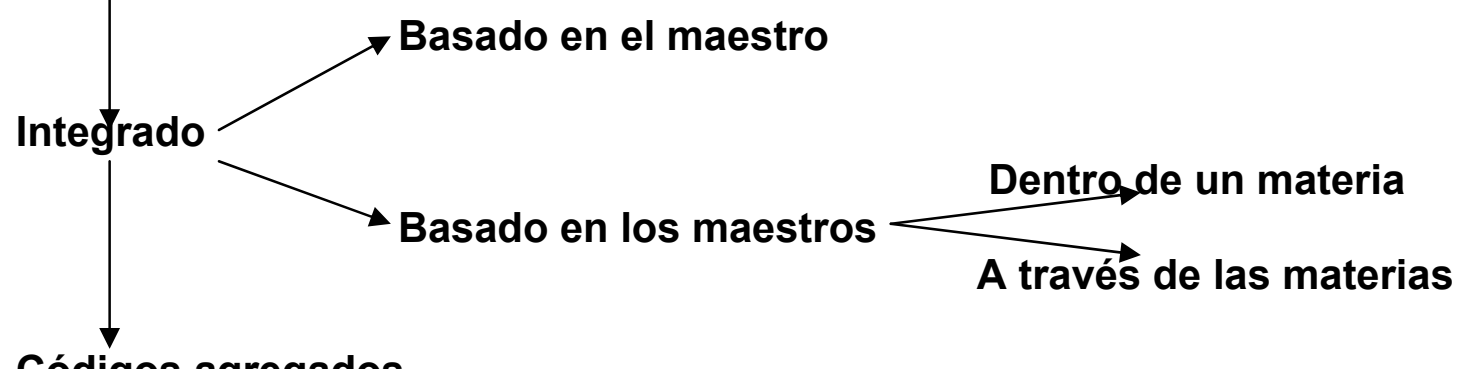

Códigos agregados

La primera gran distinción que se puede establecer dentro de los códigos agregados se efectúa entre tipos especializados y tipos no especializados. La extensión de la especialización puede medirse en términos del número de contenidos cerrados públicamente examinados al fin de la etapa educativa secundaria. Así, en Inglaterra, a pesar de que no hay un límite formal, el estudiante generalmente presenta en el examen tres materias de nivel "A" en comparación con el número mayor de materias que se deben presentar en Alemania para obtener el "Abitur", en Francia para obtener el "Baccalaureat", o en Suecia para obtener el "Studente Exam".

Dentro del tipo especializado inglés, podemos distinguir dos variedades: la pura y la impura. La variedad pura existe cuando las materias de nivel " $A$ " se extraen de un universo común de conocimiento, por ejemplo, Química, Física, Matemáticas. La variedad impura existe cuando las materias del nivel "A" se extraen de diferentes universos de conocimiento, por ejemplo, Religión, Física, Economía. Esta última combinación, a pesar de que es formalmente posible, muy raras veces existe sustantivamente, pues los alumnos no son estimulados a presentar — ni el horario es usualmente posible - tal combinación. Es interesante observar que hasta hace poco la variedad pura en la universidad (nivel universitario) recibía el esta-tus más alto de un grado con honores, mientras que la variedad impura se orientaba hacia los grados generales de bajo estatus $^{21}$. Uno puede detectar los comienzos de un cambio en Inglaterra de la variedad

${ }^{21}$ Considérese el acalorado debate sobre el intento de obtener permiso de Oxford para desarrollar carreras en Antropología, Sociología, Psicología y Biología - una combinación relativamente "pura". 
pura a la variedad impura, la cual parece estar tratando de orientarse hacia el tipo no especializado del código agregado.

Dentro del código agregado no-especializado, podemos distinguir dos variedades de acuerdo a si la unidad básica de conocimiento es la materia o el curso. Así, la forma europea estándar del código agregado es la forma no especializada, basada en la materia. La forma del código agregado en los Estados Unidos es no-especializada, basada en el curso.

Hasta aquí hemos descrito los sub-tipos y variedades de código agregado en términos descriptivos simples; en consecuencia no es fácil ver cómo sus rasgos distintivos pueden ser traducidos en conceptos sociológicos con el objeto de dar cuenta de un problema sociológico específico. De manera más clara, el lenguaje conceptual desarrollado aquí ha sido construido dentro de una perspectiva específica: la del poder y el control social. En el proceso de traducción de los rasgos descriptivos al lenguaje de la clasificación y enmarcación, es necesario preguntarse si la hipótesis acerca de sus fuerzas relativas encajan en casos particulares.

A manera de ilustración se presentan a continuación algunas hipótesis:

1) Sugerimos que la forma europea de código agregado especializado y basado en la materia, envuelve clasificación fuerte y enmarcación excepcionalmente fuerte. Esto es, en los niveles inferiores a la educación superior, existen relativamente pocas opciones disponibles para el maestro y especialmente para el alumno en relación con la transmisión del conocimiento. Los currícula y los programas son muy explícitos.

2) Sugerimos que la versión inglesa envuelve de manera excepcional una clasificación fuerte, pero una enmarcación relativamente débil en comparación con el tipo europeo. El hecho de que el tipo sea especializado determina qué contenidos (materias) deben agruparse. Existe un aislamiento muy fuerte entre el conocimiento "puro" y el conocimiento "aplicado". Los currícula se gradúan para grupos de habilidades particulares. Puede haber un alto aislamiento entre una materia y una clase de alumnos. En la escuela secundaria, por ejemplo, los alumnos del grupo " $\mathrm{D}$ " no tienen acceso a ciertas materias. Lo mismo sucede con los alumnos del grupo 'A' $X$ Sin embargo, sugerimos que la enmarcación, en relación con Europa, es débil. Esto puede observarse particularmente en el nivel primario. Existe también, en relación con Europa, menos control central sobre lo que se transmite, aunque, sin embargo, los diversos requisitos del nivel universitario ejercen un control fuerte sobre la escuela secundaria ${ }^{22}$. Aunque esto es relativo, existe una enmarcación más débil en Inglaterra entre el conocimiento educativo y el conocimiento cotidiano comunitario para cierta clase de estudiantes: los llamados menos capaces (less able). Finalmente, en relación con Europa, sugerimos que hay más opciones disponibles al estudiante en la relación pedagógica. La enmarcación referida a los alumnos es débil. De esta manera podemos considerar que la enmarcación referida a la relación maestro-alumno es relativamente débil pero la

\footnotetext{
${ }^{22}$ El contenido de los exámenes públicos entre la escuela secundaria y la universidad está controlado directa o indirectamente por la universidad mediante el control sobre los diferentes syllabuses. Así, si va a haber un cambio en las syllabuses y currícula de la escuela secundaria, esto requiere cambios en la política del nivel terciario ya que esto afecta el ingreso de los estudiantes. Tal cambio en la política universitaria incluiría cambios en la selección, organización, ritmo y temporalización del conocimiento en el nivel terciario. De esta manera, las condiciones para un cambio en el código del conocimiento en el nivel secundario están constituidas por un cambio en el nivel terciario. Cambios en el código del conocimiento en el nivel secundario probablemente sea de naturaleza limitada si no ocurren cambios similares en el nivel terciario. Existen, por supuesto, otros grupos (la industria) cuyo interés puede afectar un curriculum y syllabuses dados.
} 
clasificación es relativamente mucho más fuerte en el sistema inglés que en el sistema europeo. Escocia está mucho más cerca de la versión europea del código agregado.

3) En los Estados Unidos la forma no-especializada del código agregado basado en el curso tiene la clasificación y enmarcación más débiles del código agregado, especialmente en el nivel secundario y universitario. Una gran cantidad de materias pueden tomarse tanto en el nivel secundario como en la universidad y pueden realizarse diferentes combinaciones.

Esto indica una clasificación débil. El aislamiento entre el conocimiento educativo y el conocimiento cotidiano comunitario es débil, como puede evidenciarse en el control de la comunidad sobre la escuela; esto indica enmarcaciones débiles. El número de opciones disponibles a los alumnos dentro de la relación pedagógica es amplio. Podríamos suponer que la clasificación y la enmarcación, en los Estados Unidos, es la más débil de los códigos agregados.

\section{Códigos integrados}

Es importante tener claro el término "integrado". El hecho de que una materia use las teorías de otra materia, no quiere decir, que este tipo de relación intelectual constituya integración. Tal inter-relación intelectual puede muy bien formar parte de un código agregado y darse sólo en ciertos momentos en la historia del desarrollo del conocimiento. La noción de "integración" tal como se usa aquí, se refiere mínimamente a la subordinación de materias o cursos, previamente aislados, a una idea que los relaciona, lo cual borra la delimitación entre las materias. Podemos distinguir dos tipos de integración. El primer tipo, basado en el maestro. Aquí, el maestro, como sucede en la escuela infantil, tiene una amplia cantidad de tiempo, a menudo con el mismo grupo de alumnos. El maestro puede operar con un código agregado y mantener las diversas materias diferenciadas y aisladas, o puede borrar la delimitación entre ellas. Este tipo de código integrado es más fácil de introducir que el segundo tipo, el cual está basado en los maestros. Aquí, la integración envuelve relaciones con otros maestros. En esta forma, podemos tener diversos grados de integración en términos del número de maestros envueltos.

Podemos, además, distinguir dos variedades de integración, teniendo en cuenta si ésta se refiere a un grupo de maestros dentro de una materia común o si envuelve maestros de diferentes materias. Los códigos integrados, aunque por definición, poseen una clasificación débil, pueden variar en la enmarcación. Durante el período de iniciación, la enmarcación en la cual entra el maestro será débil, pero diferentes factores afectarán la fuerza de la enmarcación final. Es también posible que la enmarcación en la cual entran los alumnos pueda variar en su fuerza.

De esta manera, los códigos integrados pueden estar confinados a una materia o ellos pueden cruzar diferentes materias. Podemos hablar de la fuerza del código en términos del número de materias diferentes coordinadas por el código, o, si este criterio no puede aplicarse, podemos medir la fuerza del código en términos del número de maestros que éste coordina.

Los códigos integrados pueden variar la fuerza de la enmarcación en la medida en que ésta se aplica a los maestros o a los alumnos.

Desde la perspectiva desarrollada aquí, las diferencias dentro de, y entre los códigos del conocimiento educativo, yacen en las variaciones de la fuerza y en la naturaleza de 
los procedimientos para mantener los límites en la medida en que éstos son dados por la clasificación y enmarcación del conocimiento. Puede verse, que la naturaleza de la clasificación y de la en-marcación afecta la estructura de poder-autoridad que controla la diseminación del conocimiento educativo, y la forma del conocimiento transmitido. En esta forma los principios de poder y de control social se realizan a través de los códigos del conocimiento educativo $y$, a través de dichos códigos, entran en, y configuran la conciencia. De esta manera, las variaciones dentro de, y el cambio de los códigos del conocimiento debieran ser un problema crítico de interés para los sociólogos. Este análisis plantea los siguientes problemas:

1) Cuáles son los antecedentes de las variaciones en la fuerza de la clasificación y de la enmarcación? ${ }^{23}$

2) Cómo una estructura con una clasificación y enmarcación dadas se perpetúa a sí misma? Cuáles son las condiciones del cambio y las resistencias a este?

3) Cuáles son las diferentes experiencias socializantes realizadas a través de las variaciones en la fuerza de las clasificaciones y de las enmarcaciones?

Limitaremos la aplicación de este análisis a la consideración de los aspectos de las dos últimas preguntas. Pienso que debo pedir excusas al lector por este largo y tal vez pesado, análisis conceptual, antes de haberle dado una noción del punto de vista al cual dirige.

\section{Aplicación}

A continuación examinaremos los patrones de relación social y las consecuencias socializantes, que se realizan a través de la versión europea-particularmente la inglesadel código agregado, y aquellas que, se espera, surgen de los códigos integrados, particularmente de aquellos que desarrollan una enmarcación débil. Plantearemos que existe cierto movimiento hacia las formas del código integrado y examinaremos la naturaleza de la resistencia a tal cambio. Igualmente, plantearemos algunas razones para este cambio.

\section{Clasificación y enmarcación de la forma europea del código agregado}

Este análisis presentará algunas dificultades ya que en algunas ocasiones pasaremos del nivel secundario al nivel universitario. A pesar de que el sistema inglés presenta como rasgo distintivo la especialización, comparte ciertos rasgos del sistema europeo. Teniendo en cuenta que éste es el comienzo de una teoría sociológica limitada que explora la organización y la estructuración social del conocimiento, es claro que todos los planteamientos, inclusive aquellos que tienen un carácter descriptivo, son hipotéticos. Los planteamientos descriptivos han sido organizados selectivamente de acuerdo con el significado que tienen para la teoría.

Una de las mayores diferencias entre las versiones europea e inglesa del código agregado consiste en que, con el tipo especializado inglés, se establece una categoría de pertenencia muy temprano en la carrera educativa, en términos de una escogencia temprana entre lo puro y lo aplicado, entre las ciencias y las artes, entre la adquisición y la

\footnotetext{
${ }^{23}$ Tales variaciones pueden muy bien estar ligadas a variaciones en el desarrollo de la estructura de clase. Véase, "Clases sociales y pedagogías..." (Publicado en este número).
} 
no adquisición de una identidad educativa específica. El estatus particular en un código agregado dado se hace claro mediante la selección de los niños de una misma edad en diferentes grupos, o mediante un sistema sofisticado de gradación. En este caso, casi siempre se conoce el significado social del lugar donde se está, y en particular, de quién se es, con cada avance en la carrera educativa. (Inicialmente, estoy haciendo ciencias, o artes pura o aplicadas; o no estoy haciendo nada; más tarde me convierto en un físico, un economista, un químico, etc.). La lealtad a la materia se desarrolla sistemáticamente, con cada avance en la vida educativa, en los alumnos primero y finalmente en los estudiantes, y se transmite tanto por maestros como por profesores. El sistema se autoperpetúa a través de esta forma de socialización. Con la forma especializada del código agregado es banal decir que en la medida en que uno se vuelve más viejo aprende más y más acerca de menos y menos. Otra forma más sociológica de plantear lo mismo consiste en decir que en la medida en que uno se vuelve más viejo, se hace progresivamente diferente de los demás. Es claro que esto sucede en algún momento de la carrera educativa, pero con la especialización, esto sucede muy pronto. Por lo tanto, la especialización muy pronto revela la diferencia de en lugar de la comunalidad con. La especialización crea relativamente rápido una identidad educativa que está claramente marcada y delimitada. La categoría o identidad educativa es pura. Las versiones especializadas del código agregado tienden a eliminar categorías mixtas e identidades borradas, ya que éstas representan una apertura potencial, y una ambigüedad que produce consecuencias problemáticas a la socialización previa. Las categorías mixtas tales como la de bio-físico, psico-lingüista, se permiten desarrollar sólo después de una larga socialización dentro de la lealtad a una materia. De esta manera, para cambiar una identidad, tiene que debilitarse la identidad anterior y crearse una nueva identidad. Por ejemplo, en Inglaterra, si un estudiante tiene un primer grado en psicología y desea continuar un postgrado en sociología, o no se le permite hacer el cambio, o si se le permite, éste debe presentar un cierto número de exámenes a nivel de grado inicial en sociología. En el proceso de preparación de estos exámenes, el estudiante usualmente entra en un proceso de relaciones sociales con sociólogos acreditados y con estudiantes mediante los cuales adquiere el estilo cognitivo y social particular a la identidad sociológica. El cambio en la identidad educativa se cumple mediante un proceso de re-socialización dentro de una nueva lealtad a una materia. El sentido de lo sagrado, el sentido de diferencia del conocimiento educativo no surge tanto de una ética del conocimiento en si mismo, sino que es más bien una función de la socialización en una lealtad a una materia, pues es la materia la que se convierte en el eje de la identidad. Cualquier intento por debilitar o por cambiar la fuerza de la clasificación (o aún, la fuerza de la enmarcación) puede sentirse como una amenaza a la identidad propia y puede experimentarse como una contaminación que hace peligrar lo sagrado. Tenemos aquí una fuente de resistencia al cambio del código educativo.

La versión especializada del código agregado desarrolla procedimientos cuidadosos de selección para ver quién pertenece y quien no pertenece, y una vez tal selección ha tenido lugar, es muy difícil cambiar una identidad educativa. Las diferentes clases de conocimiento están marcadamente aisladas entre sí. La selección y la diferenciación son los rasgos tempranos de este código particular Así, la estructura profunda del tipo especializado del código agregado es el mantenimiento de la delimitación fuerte que produce el control desde dentro, a través de la formación de identidades específicas. Esto constituye un interesante aspecto del espíritu protestante.

El mantenimiento de la delimitación fuerte puede ilustrarse con referencia al intento por institucionalizar nuevas formas o a los empeños para cambiar la clasificación fuerte dentro del tipo de código agregado europeo, o dentro del inglés. A causa de la fuerza 
excepcional de la clasificación en Inglaterra, las dificultades son mayores allí. Los cambios en la fuerza de la clasificación y la institucionalización de nuevas formas de conocimiento pueden convertirse en un asunto de importancia cuando hay cambios en la estructura del conocimiento en los niveles más altos y/o cambios en la economía. Con la aparición de nuevas formas de conocimiento surgen problemas críticos tales como el de su legitimidad, a qué instancia las formas pertenecen, cuándo, dónde y por quiénes tales formas deben ser enseñadas. Nos hemos referido a lo "sagrado" en términos de una identidad educativa, aun, cuando también existe el aspecto "profano" del conocimiento. Podemos considerar como "profano" el aspecto de la propiedad del conocimiento. Cualquier nueva forma o debilitamiento de la clasificación claramente se deriva de clasificaciones pasadas. Tales formas nuevas o clasificaciones debilitadas pueden mirarse como intentos para romper o debilitar monopolios existentes. El conocimiento bajo un código agregado se considera propiedad privada con su propia estructura de poder y situación de mercado. Esto afecta todo el ambiente que rodea el desarrollo y el mercado del nuevo conocimiento. Los niños y los alumnos son socializados muy temprano en este concepto de conocimiento como propiedad privada. Ellos son estimulados a trabajar como individuos aislados con sus propios instrumentos alrededor de su trabajo. Este fenómeno, hasta hace poco, podía ser observado en las"grammar schools". También puede ser observado más claramente en los salones de examen. Los alumnos y los estudiantes particularmente en las artes, aparecen, desde este punto de vista, como cierto tipo de empresarios.

Existen, entonces, fuertes controles internos sobre la institucionalización de nuevas formas de conocimiento, sobre el cambio de la fuerza de la clasificación y sobre la producción de nuevo conocimiento que deriva tanto de fuentes "sagradas" como "profanas".

Hasta aquí hemos considerado las relaciones entre la clasificación fuerte del conocimiento, el concepto de la propiedad y la creación de identidades específicas con referencia particular a la forma especializada del código agregado. Ahora pasaremos de la clasificación del conocimiento a su enmarcación en el proceso de transmisión.

Cualquier código agregado envuelve una organización jerárquica del conocimiento de tal forma que el último misterio de la materia se revela muy tarde en la vida educativa. Por el último misterio de la materia, significamos su potencialidad para crear nuevas realidades.

Se da también el caso, y esto es importante, que el último misterio de la materia no es coherente, sino incoherente: no es el orden sino el desorden, no es lo conocido si no lo desconocido. En la medida en que este misterio, bajo los códigos agregados, se revela muy tarde en la vida educativa $-y$ sólo a unos pocos seleccionados que han mostrado signos de socialización exitosa- entonces solo unos pocos experimentan en su interior la noción de que el conocimiento es permeable, que su ordenamiento es provisional, que la dialéctica del conocimiento es la del cierre y la apertura. Para la mayoría la socialización en el conocimiento es la socialización en el orden, el orden existente, en la experiencia de que el mundo del conocimiento educativo es impermeable. Tenemos aquí otra versión de alienación?

La historia de cualquier forma de conocimiento educativo muestra precisamente el poder de tal conocimiento para crear, indefinidamente nuevas realidades. Sin embargo, la socialización en la enmarcación específica del conocimiento en su transmisión puede hacer tal historia, para la experiencia, algo sin significado. El concepto clave del código 
agregado europeo es la disciplina. Esto significa aprender a trabajar dentro de un marco recibido. Significa, igualmente aprender qué preguntas pueden hacerse en un momento particular. A causa del ordenamiento jerárquico del conocimiento en el tiempo ciertas preguntas no entran en un mareo particular.

Esto se aprende muy pronto tanto por el maestro como por los alumnos Disciplina, entonces, significa aceptar una selección dada, una organización, un ritmo y una temporalización del conocimiento realizada en el marco pedagógico. Con el incremento en la vida educativa, hay un debilitamiento progresivo de la enmarcación tanto para el maestro como para el alumno. Sólo aquellos pocos que han mostrado signos de socialización exitosa tienen acceso a marcos relajados. Para la masa de la población la enmarcación es fuerte. En cierta forma, la forma europea del código agregado pone a salvo el conocimiento mediante el proceso de socialización en sus enmarcaciones. Existe una tendencia, que varía con la fuerza de la en-marcación específica, a socializar a los jóvenes en principios asignados, en operaciones rutinarias y en sus derivaciones. El sistema evaluativo hace énfasis sobre la adquisición de estados de conocimiento antes que sobre las formas de conocer. Un estudio de las preguntas y formatos de exámenes, de la estructura simbólica de la evaluación, sería desde este punto de vista, un importante estudio empírico. De esta manera, el conocimiento, tiende a ser transmitido, particularmente a alumnos de la élite en el nivel secundario, mediante enmarcaciones fuertes que controlan la selección, organización, ritmo ${ }^{24}$ y temporalización del conocimiento. La adquisición del conocimiento no es tanto un derecho como algo que debe ser ganado o trabajado. Entre más fuertes sean la clasificación y la enmarcación, más tiende la relación educativa a ser jerarquizada y ritualizada, en tanto que el estudiante es visto como un ignorante con muy poco estatus y pocos derechos. Estas son cosas que uno gana más bien como una recompensa y que se usan con el objeto de entusiasmar y mantener la motivación de los alumnos. Dependiendo de la fuerza de la enmarcación el conocimiento se transmite en un contexto donde el maestro tiene el máximo control o vigilancia, como sucede en las relaciones jerarquizadas de la escuela secundaria.

Podemos mirar el problema de la enmarcación del conocimiento en la relación pedagógica desde otro punto de vista, En cierta forma, el conocimiento educativo es conocimiento que no pertenece al conocimiento del sentido común. Es conocimiento liberado de lo particular, de lo local mediante los diversos lenguajes de las ciencias o formas de reflexividad de las artes que hacen posible tanto la creación como el descubrimiento de nuevas realidades. Esto plantea inmediatamente la pregunta de la relación entre el conocimiento no-común de la escuela y el conocimiento común, el conocimiento diario, de la comunidad, del alumno, de su familia y de los grupos de jóvenes. Esta formulación nos invita a preguntar qué tan fuertes son los marcos del conocimiento educativo en relación con el conocimiento experiencial, basado en la comunidad, esto es, no-escolar? Sugerimos que los marcos del código agregado, desde

\footnotetext{
${ }^{24}$ Lo que siempre se subestima es el hecho de que el ritmo del aprendizaje esperado implícitamente se base sobre la socialización que la clase media hace del niño. La socialización del niño en las familias de la clase media constituye una ayuda oculta en el sentido en que la familia provee un ambiente físico y psicológico que facilita inmensamente, en diferentes formas, el aprendizaje en la escuela. El niño de la clase media está orientado hacia el aprendizaje de casi todas las cosas. A causa de esta ayuda oculta, ha habido pocos incentivos para cambiar el curriculum y la pedagogía, pues el niño de la clase media está muy cerca del aprendizaje. Puede que no le cueste o que no apruebe lo que aprende, pero él aprende Cuando la escuela (el sistema de la escuela) no está subsidiado por el hogar el niño a menudo fracasa. En esta forma, aún el ritmo del conocimiento educativo está basado en las clases sociales. Es probable que la fuerza del enmarcamiento en la media en que se refiere al ritmo constituya una variable crítica en el estudio de la educabilidad. Es posible que el enmarcamiento débil de los códigos integrados (con referencia al ritmo) indique que estos códigos presuponen un promedio de vida educativa más largo. Los niños de la clase media pueden ser o haber sido alumnos potenciales para las escuelas progresivas a causa de su larga vida educativa.
} 
los más tempranos años de la infancia socializan el niño en marcos de conocimiento que desaniman cualquier conexión con las realidades diarias, o que producen una red altamente selectiva para establecer la conexión. Mediante tal socialización el niño pronto aprende qué es lo que puede traer del exterior a la relación pedagógica. Tal enmarcación también hace del conocimiento educativo algo no ordinario o mundano sino esotérico que da un significado especial a aquellos que lo poseen. Es posible pensar que cuando este marco se relaja para incluir realidades cotidianas, es a menudo y algunas veces válido, no sólo para la transmisión del conocimiento educativo, sino también para propósitos de control social de formas de delincuencia. El debilitamiento de este marco ocurre usualmente con los niños "menos capaces' a quienes se les ha impedido la educación.

En general, y dependiendo de la fuerza específica de la clasificación y de la enmarcación, la forma europea del código agregado es rígidamente diferenciadora y jerárquica en carácter; además, es altamente resistente al cambio, particularmente, en el nivel secundario. Con la versión inglesa del código agregado, esta resistencia al cambio aumenta por la autoridad que se otorga a los directores y a los principals*. En Inglaterra dentro de las restricciones del sistema de exámenes públicos, los directores de escuelas tienen una libertad relativamente amplia sobre la organización y la transmisión del conocimiento. El control central sobre el código educativo es relativamente débil en Inglaterra, a pesar de que las escuelas están sujetas a la inspección tanto de las autoridades del gobierno central como del local. Sin embargo, la relación entre el cuerpo de inspectores y las escuelas es muy ambiguo. Para producir un amplio cambio en Inglaterra se requería la participación de cientos de escuelas individuales. De esta manera, observamos que la rigidez en los códigos del conocimiento educativo pueden surgir tanto de su control centralizado como de su control central débil El control central débil permite una serie de cambios que tienen inicialmente, consecuencias limitadas para el sistema en su conjunto. Por otra parte, existe un control central mucho más fuerte sobre el estilo organizativo de la escuela. Esto puede conducir a una situación en la cual puede haber un cambio en el estilo organizativo sin que haya un cambio marcado en el código del conocimiento educativo, particularmente donde el código educativo en sí mismo crea identidades específicas. Esto plantea el problema, que no puede ser resuelto aquí, de las relaciones entre el cambio organizativo y el cambio en el código del conocimiento educativo, esto es, el cambio en la fuerza de la clasificación y de la enmarcación.

Tenemos entonces que, las formas europeas e inglesa del código agregado pueden proporcionar, para aquellos que van más allá del estado de iniciación, orden, identidad y aceptación. Para aquellos que no pasan este estado, la forma del código puede ser penosa, hiriente, y carecer de sentido. Esto es lo que Bourdieu llama "la violencia simbólica".

\section{Códigos agregados e integrados}

Examinaremos ahora una forma de código integrado que se realiza a través de clasificación y enmarcación débiles. En el curso de este análisis presentaremos aspectos adicionales sobre los códigos integrados.

Existe un número de intentos para institucionalizar las formas del código integrado en diferentes modalidades, sobre todo a nivel de la educación pre-escolar. El proyecto "Nuffield Science" constituye un intento de realizar la integración con las ciencias físicas, y el Centro Chelsea para la Educación Científica, el Centro Universitario de Tecnología

\footnotetext{
${ }^{*}$ Se denomina principals a los directores de algunas universidades, colleges y escuelas. (Nota del traductor).
} 
Chelsea de la Universidad de Londres, están dedicados casi completamente al entrenamiento de estudiantes en esta aproximación. La señora Charity James, del Goldsmith's College de la Universidad de Londres está también realizando cursos de entrenamiento para las formas del código integrado. Un gran número de escuelas comprensivas están experimentando con esta aproximación en el nivel de la escuela media. EI SDS en Alemania, y diferentes grupos de estudiantes radicales, están explorando este tipo de código con el propósito de usar los medios de la universidad contra su significado. Sin embargo, probablemente es cierto decir que el código en este momento existe a nivel de la ideología y de la teoría que existe sólo en un número relativamente pequeño de escuelas y agencias educativas que intentan institucionalizarlo con seriedad.

Como dijimos al principio de este artículo, con el código integrado tenemos el cambio de un contenido cerrado a un contenido abierto, de una clasificación marcada a una clasificación reducida. Inmediatamente podemos observar que esta alteración en la clasificación del conocimiento conduce a una alteración en las estructuras de autoridad existentes, en las identidades educativas específicas y en los conceptos de propiedad.

Cuando tenemos integración, los diversos contenidos están subordinados a alguna idea que reduce su aislamiento entre sí. De esta manera, la integración reduce la autoridad de contenidos separados y esto tiene implicaciones para las estructuras de autoridad existentes. Cuando tenemos un código agregado, éste permite, en principio, considerables diferencias en la pedagogía y en la evaluación, a causa del alto aislamiento entre los diferentes contenidos. Sin embargo, la autonomía del contenido constituye el otro lado de una estructura que ejerce una supervisión extremadamente celosa. Es posible sugerir que el código integrado no permite variaciones en la pedagogía y en la evaluación las cuales son posibles dentro de los códigos agregados. Por el contrario, existe una marcada tendencia hacia una pedagogía común y hacia una forma de evaluación común. En otras palabras, los códigos integrados probablemente producen a nivel de los maestros homogeneidad en la práctica de la enseñanza. Así, los códigos agregados aumentan la libertad del maestro (siempre dentro de los límites de la clasificación y enmarcación existentes) mientras que los códigos integrados reducen la libertad del maestro en una relación directa con la fuerza del código integrado (número de maestros-coordinados por el código). Por otra parte, se puede argumentar que la libertad creciente de los maestros dentro de los códigos agregados es paralela a la reducción de la libertad de los alumnos y que la reducida libertad de los maestros dentro de los códigos integrados es paralela a la creciente libertad de los alumnos. En otras palabras, hay un cambio en el balance del poder entre el maestro y el alumno en la relación pedagógica.

Desarrollaremos a continuación estos puntos. Con el objeto de cumplir cualquier forma de integración (como algo que se diferencia del enfoque de las diferentes materias sobre un problema común, lo cual da origen a lo que podríamos llamar un curriculum "enfocado") debe existir alguna idea relacionante, o un concepto superior a un contenido, el cual se enfoca sobre principios generales a un alto nivel de abstracción. Por ejemplo, si las relaciones entre la sociología y la biología van a ser abiertas, entonces la idea relacionante (entre otras) puede ser el aspecto de los problemas del orden y del cambio examinados a través de los conceptos de códigos genético y cultural. Cualquiera que sean los conceptos relacionantes, éstos actuarán selectivamente sobre el conocimiento dentro de cada materia que se va a transmitir.

Las particularidades de cada materia probablemente tengan en este caso, un significado reducido. El concepto relacionante enfocará su atención sobre la estructura 
profunda de cada materia antes que sobre su estructura superficial. Esto conducirá a efectuar un énfasis sobre, y una exploración de, los principios generales y los conceptos mediante los cuales tales principios son obtenidos. A su vez, esto probablemente afecte la orientación de la pedagogía, la cual se concentrará menos en la necesidad de adquirir estados de conocimiento y más en cómo el conocimiento se produce. En otras palabras, la pedagogía de los códigos integrados hace mucho énfasis en las diversas formas de conocer en la relación pedagógica. Con el código agregado la pedagogía tiende a proceder de la estructura superficial del conocimiento a su estructura profunda, y como hemos visto, sólo la élite tiene acceso a la estructura profunda y por lo tanto acceso a la manifestación de nuevas realidades, o acceso al conocimiento vivencial que las nuevas realidades hacen posible. Con los códigos integrados, la pedagogía generalmente procede de la estructura profunda a la estructura superficial. La realización de este aspecto puede verse en el trabajo que en matemáticas realiza la nueva escuela primaria. Se puede considerar que los códigos integrados hacen accesible al alumno, desde el comienzo de su carrera educativa, la estructura profunda del conocimiento, esto es, hacen accesibles los principios para la generación de nuevo conocimiento, en una forma apropiada a un cierto nivel de edad. El énfasis en las diversas formas de conocer antes que sobre la adquisición de estados de conocimiento, probablemente afecte no sólo la pedagogía sino también la teoría subyacente del aprendizaje. La teoría del aprendizaje que subyace a un código agregado es generalmente didáctica, mientras que la teoría del aprendizaje que subyace a los códigos integrados se basa más en el grupo, y es más autoregulativa. Esto surge de la diferencia conceptual que se da en relación con lo que cuenta como adquisición de conocimiento, lo cual a su vez, conduce a una conceptualización diferente acerca de cómo el conocimiento va a ser adquirido. Estos cambios en el énfasis y en la orientación de la pedagogía son, inicialmente, responsables del relajamiento de los marcos en los cuales entran el maestro y los alumnos. Los marcos relajados no sólo cambian la naturaleza de las relaciones de autoridad mediante el incremento de los derechos del educando, sino que también pueden debilitar o borrar la delimitación entre lo que puede y lo que no puede ser enseñado. De esta manera son mayores los aportes del maestro y del alumno que entran en la relación pedagógica. La lógica inherente al código integrado probablemente cree un cambio en la estructura de los grupos de alumnos, los cuales pueden exhibir una considerable flexibilidad. El concepto de mantenimiento relativamente débil de la delimitación que constituye el eje central de los códigos integrados se realiza tanto en la estructuración del conocimiento educativo como en la organización de las relaciones sociales.

Introduciremos ahora algunas consecuencias organizativas de los códigos agregados e integrados las cuales hacen explícita la diferencia en la distribución del poder y los principios de control inherentes a estos códigos educativos.

Cuando el conocimiento está regulado mediante un código agregado, dicho conocimiento se organiza y distribuye mediante la jerarquización de materias aisladas. Esta estructura apunta hacia el control oligárquico de la institución, mediante reuniones formales e informales de los jefes de Departamento con la autoridad máxima de la institución. De esta manera, el personal docente más viejo tendrá relaciones horizontales de trabajo fuertes (esto es, con sus colegas en otra jerarquía de materias) y relaciones verticales de trabajo fuertes dentro de su propio departamento. Sin embargo, el personal docente más joven probablemente tenga sólo relaciones de trabajo y de subordinación verticales (dentro de la jerarquía de materias).

La subordinación del personal docente joven es vertical en lugar de ser horizontal por las siguientes razones. Primero, el personal docente ha sido socializado dentro de una 
lealtad muy fuerte a la materia y, a través de esto, dentro de identidades específicas. Estas identidades específicas se refuerzan continuamente mediante la interacción social dentro de cada departamento y mediante el aislamiento entre departamentos. Segundo, los departamentos se encuentran a menudo en relaciones de competencia para la obtención de recursos estratégicos para la enseñanza. Tercero, la promoción dentro de la jerarquía de materias a menudo descansa en la expansión de éstas. Las relaciones horizontales del personal docente joven, (particularmente, donde no existe una efectiva estructura administrativa participativa) generalmente se limitan a contactos extralaborales. Pueden existir discusiones sobre problemas de control (" $X$ del grado $3 b$ es un------ Cómo lo maneja usted?" o "No puedo hacer que X escriba una hoja").

Así pues, el código agregado dentro del marco de control oligárquico crea para el personal docente más viejo fuertes relaciones horizontales y verticales; mientras que las relaciones de trabajo del personal docente mas joven posiblemente son verticales en tanto que las relaciones horizontales están limitadas a contactos que nada tienen que ver con la actividad de trabajo. Este tipo de sistema organizativo estimula el chisme, la intriga y genera una teoría secreta de las formas de organización, pues tanto las prácticas administrativas como los actos de la enseñanza son invisibles a la mayoría del cuerpo docente.

El código integrado requiere que los maestros de materias diferentes entren en relaciones sociales que no surjan de áreas externas a las tareas docentes sino de tareas educativas compartidas y cooperativas. En esta forma el centro de gravedad de las relaciones entre los maestros sufre un cambio radical. Así, en vez de que los maestros y profesores estén divididos y aislados mediante la subordinación a jerarquías de materias, las condiciones para su unificación se dan a través de una situación de trabajo común. La transformación de la base de las relaciones entre maestros o entre profesores puede tender a debilitar las jerarquías separadas del código agregado. Estas nuevas relaciones horizontales del trabajo entre maestros o entre profesores puede alterar tanto la estructura como la distribución del poder regulada por el código agregado. Además, las prácticas administrativas y los actos específicos de la enseñanza probablemente cambien de una relativa invisibilidad a la visibilidad.

Podemos esperar desarrollos similares a nivel de los estudiantes y aun de los alumnos más avanzados. Tanto para los alumnos como para los estudiantes, cada paso en la vida educativa significa igualmente una subdivisión y un aislamiento educativo. Alumnos y estudiantes están igualmente ligados a jerarquías de materias y por razones similares, ligados al personal docente; sus identidades y su futuro están configurados por el departamento. Su subordinación vertical y sus relaciones basadas en el trabajo académico son fuertes, mientras que sus relaciones horizontales se limitan a actividades no relacionadas con el trabajo académico, (sociedades de alumnos o estudiantes) o a actividades periféricas de administración. Aquí, nuevamente, tenemos otro ejemplo de la fuerza de la delimitación de los códigos elaborados, esta vez entre áreas de trabajo y áreas de ningún trabajo. Los códigos integrados pueden, muy bien, proporcionar las condiciones para que existan relaciones sociales y de subordinación horizontales entre los estudiantes y entre los alumnos basadas en tareas comunes (transmisión y recepción del conocimiento) ${ }^{25}$.

\footnotetext{
${ }^{25}$ Es posible que los procedimientos de mantenimiento de los límites débiles de los códigos integrados en el nivel de la estructura organizativa, la estructura del conocimiento y la estructura de identidad puedan incrementar las afiliaciones de los alumnos/estudiantes a grupos informales de edad como una fuente de identidad, relación y organización.
} 
En esta situación, es posible esperar un debilitamiento de la delimitación entre el personal docente, especialmente el joven, y los alumnos/estudiantes.

Así, un movimiento desde los códigos agregados hacia los códigos integrados puede crear alteraciones en la estructura y en la distribución del poder, en las relaciones de propiedad y en las identidades educativas existentes. Este cambio del código educativo envuelve un cambio fundamental en la naturaleza y fuerza de las delimitaciones. También envuelve un cambio en lo que cuenta como posesión del conocimiento, en lo que cuenta como válida transmisión del conocimiento y un cambio en el contexto organizativo. A nivel cultural, envuelve una transformación del mantenimiento de categorías puras a la mezcla de categorías. A nivel de socialización, los resultados de los códigos integrados podrían ser menos predecibles que los resultados de los códigos agregados. Este cambio de código envuelve cambios fundamentales en la clasificación y enmarcación del conocimiento y de esta manera cambios en la estructura y distribución del poder y en los principios de control. No hay duda que el cambio de los códigos educativos genera profundas resistencias.

\section{Los códigos agregados, los códigos integrados y los problemas del orden}

Nos referiremos ahora a los aspectos relacionados con los problemas del orden. Cuando el conocimiento está regulado por códigos agregados, el orden social surge de la naturaleza jerárquica de las relaciones de autoridad, del ordenamiento sistemático del conocimiento diferenciado en el tiempo y en el espacio, y de unos procedimientos de examen, explícitos, y usualmente precedibles El orden interno al individuo se crea mediante la formación de identidades específicas. La expresión institucional de la clasificación y de la enmarcación fuertes crea la predictibilídad en el tiempo y en el espacio. Debido a la clasificación fuerte los códigos agregados permiten una gran cantidad de variaciones entre las materias en relación con la organización, transmisión y evaluación del conocimiento. Debido a la clasificación fuerte este código permite, al personal docente, en principio, sostener (dentro de ciertos límites) una gran variedad de ideologías porque los conflictos pueden estar contenidos dentro de sus diversas jerarquías aisladas. En los niveles inferiores a la universidad los fuertes límites entre el conocimiento educativo y el conocimiento no-educativo relevante, pueden, en principio, facilitar la diversidad de ideologías del personal docente ya que éstas no pueden ofrecerse explícitamente. Al mismo tiempo la enmarcación fuerte hace altamente visible cualquier intrusión. La variedad de libertades personales en el nivel de la universidad está simbolizada en el sistema ático de algunos códigos agregados y esto forma las bases para la cohesión del todo diferenciado.

Mientras usualmente se da el caso de que los códigos agregados, en relación con los códigos integrados, crean enmarcaciones fuertes entre el conocimiento no-común de la escuela y el conocimiento comunitario cotidiano del maestro y del alumno, también se da el caso que tal aislamiento crea amas de privacidad, pues, en la medida en que la experiencia basada en la comunidad es irrelevante para la relación pedagógica, los aspectos de tales experiencias que informan el yo, también son irrelevantes. Estas áreas de privacidad reducen la penetración de los procesos socializantes pues es posible distanciarse de ellos. Esto significa además que la socialización puede ser profundamente traumática, tanto para aquellos que la desean, pero que no adquieren una identidad, como pura la mayoría de aquellos para quienes la adquisición de una identidad se hace prontamente irrelevante. 
El orden creado por los códigos integrados puede ser problemático. Es posible pensar que si no se satisfacen las cuatro condiciones siguientes, entonces, la apertura del aprendizaje bajo la integración puede producir una cultura en la cual ni los maestros ni los alumnos tendrán un sentido del tiempo, lugar y propósito. A continuación comentamos estas cuatro condiciones:

1) Debe haber consenso acerca de la idea integradora y ésta debe ser suficientemente explícita, (es irónico que el movimiento hacia la integración se esté dando en aquellos países donde existe un bajo nivel de consenso moral). Puede suceder que los códigos integrados sólo funcionen ${ }^{26}$ cuando hay un alto nivel de consenso ideológico entre el personal docente. Ya hemos visto que en comparación con los códigos agregados, los códigos integrados demandan mayor homogeneidad en la pedagogía y en la evaluación y, por lo tanto, reducen las diferencias entre los maestros en cuanto a las formas de transmisión y evaluación del conocimiento. Mientras que los procesos de enseñanza bajo los códigos agregados se vuelven probablemente invisibles para los maestros, al menos que prevalezcan condiciones especiales, es posible que los procesos de enseñanza regulados por los códigos integrados sean visibles como resultado de los desarrollos de la pedagogía en la dirección de la flexibilidad en la estructura de los grupos para la enseñanza. También se da el caso de que la clasificación débil y las enmarcaciones relajadas de los códigos integrados permiten grandes expresiones de diferencia entre los maestros y posiblemente entre los alumnos en la selección de lo que se enseña. Las bases morales de las escogencias educativas son probablemente explícitas en los primeros estadios de planeación. Los códigos integrados también debilitan identidades específicas. Por las razones anteriores, los códigos integrados requieren un alto nivel de consenso ideológico, y esto puede afectar el reclutamiento del personal docente. Los códigos integrados a nivel superficial crean límites débiles o no marcados, pero a nivel profundo descansan sobre ideologías cerradas explícitas. Cuando tales ideologías no son compartidas, las consecuencias se hacen visibles y amenazan la totalidad del código a cada momento.

2) La naturaleza de la unión entre la idea relacionante y el conocimiento que se coordina debe ser también coherentemente explicitada. Es esta unión la que constituye el elemento básico para la puesta de maestros y alumnos en sus relaciones de trabajo. El desarrollo de tal marco de trabajo coordinado lo constituirá el proceso de socialización de los maestros en el código. Durante este proceso, los maestros internalizarán, como en todos los procesos de socialización, los procedimientos interpretativos del código de tal forma que dichos procedimientos se conviertan en los lineamientos implícitos que regulen y coordinen la conducta individual de los maestros en enmarcamientos relajados y en una clasificación debilitada. Esto nos conduce a una mayor distinción entre los códigos agregados y los códigos integrados. Con un código agregado el período de socialización se facilita mediante el mantenimiento de una delimitación fuerte, tanto a nivel del rol como a nivel del conocimiento. Tal socialización probablemente sea paralela a la propia socialización educativa del maestro.

Con los códigos integrados tanto el rol como la forma del conocimiento tienen que ser alcanzados en relación con una variedad de "otros" conocimientos y esto puede envolver un proceso de re-socialización si la experiencia educativa previa del maestro ha sido constituida por un código agregado. El código agregado es fácil de trabajar cuando el personal docente está constituido por maestros mediocres mientras que los códigos integrados demandan mucho mayor poder de síntesis, analogía y más

\footnotetext{
${ }^{26}$ En el sentido de la creación de orden.

Digitalizado por RED ACADEMICA
} 
habilidad tanto para tolerar como para gozar de la ambigüedad a nivel del conocimiento y de las relaciones sociales.

3) Debe constituirse un comité entre el personal docente para crear un mecanismo sensible de retro-información; el comité también actuará como agencia de socialización dentro del código. Es probable que los criterios de evaluación sean relativamente débiles en el sentido en que son posiblemente menos explícitos y mensurables que los de los códigos agregados. Como resultado, puede ser necesario desarrollar comités tanto para los maestros como para los alumnos/estudiantes, los cuales desarrollarán funciones de monitoría.

4) Una de las mayores dificultades inherentes a los códigos integrados surge de aquello que debe ser evaluado así como de la forma de evaluación, y del lugar que ocupan las competencias específicas en tal evaluación. Es probable que los códigos integrados den origen a criterios múltiples de evaluación en comparación con los códigos agregados. En el caso de los códigos agregados, dado que el conocimiento se mueve de la estructura superficial a la estructura profunda, dicho progreso crea principios ordenados de evaluación en el tiempo. La forma de cohesión temporal del conocimiento regulado mediante los códigos integrados debe determinarse previamente y hacerse explícita. Sin criterios claros de evaluación ni el maestro ni el aprendiz tienen medios para considerar el significado de lo que se aprende, ni medios para juzgar la pedagogía. En el caso de los códigos agregados, la evaluación en el nivel secundario a menudo consiste en la fusión de un escaso número de competencias específicas, estados de conocimiento y criterios previamente establecidos (que varían en su explicación) de lo que constituye una respuesta correcta, apropiada y convincente. Los criterios previamente establecidos junto al contexto social específico de evaluación producen un procedimiento relativamente objetivo. Esto no necesariamente da origen a una forma de evaluación que no tiene en cuenta los rasgos distintivos y originales de la actuación del alumno. En el caso de los códigos integrados en discusión (enmarcaciones débiles tanto para el maestro como para el alumno) dicha forma de evaluación puede ser inapropiada. Los marcos débiles habilitan una gran variedad de conductas estudiantiles para que se hagan públicas, e igualmente facilita (al menos en principio) una gran variedad entre los estudiantes. Es posible que esto conduzca a una situación en la cual la evaluación tiene más en cuenta los atributos "interiores" del estudiante. Así, sí éste tiene las actitudes "correctas", podrá alcanzar más tarde diversas competencias específicas. La actitud "correcta" puede ser evaluada en términos de la articulación entre las actitudes del estudiante y la ideología corriente. Es posible entonces, que los criterios evaluativos de los códigos integrados con enmarcamientos débiles sean débiles en la medida en que se refieren a atributos cognitivos específicos y fuertes en la medida en que se refieren a atributos disposicionales. Si esto es así, entonces una gran variedad de atributos del alumno son susceptibles de evaluación. Es también probable que una clasificación y un enmarcamiento debilitados estimulen a] alumno/estudiante a que haga más público lo suyo, haga más públicos sus pensamientos, sentimientos y valores. En esta forma más atributos del alumno son susceptibles de ser controlados. Como resultado, tendríamos que la socialización podría ser más intensiva y tal vez más penetrante. En la misma forma en que los alumnos/estudiantes se defienden contra los traumas que el código agregado proporciona, o se distancian de su código abierto, asimismo ellos pueden producir nuevas defensas contra la intrusión potencial del código integrado y sus contextos de aprendizaje abiertos. 
Podemos resumir el problema del orden como sigue. Los códigos agregados presentan rasgos fuertes y explícitos que mantienen la delimitación, y descansan sobre bases ideológicas tácitas. Los códigos integrados presentan rasgos implícitos y débiles que subvierten la delimitación y descansan sobre una base ideológica explícita y cerrada. La base ideológica del código agregado está constituida por un sistema simbólico condensado comunicado mediante sus rasgos de delimitación explícitos. Su estructura cubierta (oculta) en la solidaridad mecánica. La base ideológica de los códigos integrados no es un sistema simbólico condensado sino un sistema verbalmente elaborado y explícito. Esto constituye una realización abierta de la solidaridad orgánica que se hace sustancial mediante formas débiles de delimitación (aislamientos bajos). Es importante añadir que la estructura cubierta de solidaridad mecánica de los códigos agregados crea a través de sus "outputs" especializados la solidaridad orgánica. Por otra parte la estructura abierta de solidaridad orgánica de los códigos integrados crea a través de sus "outputs" menos especializados la solidaridad mecánica, y hace esto en la medida en que su ideología es explícita, elaborada, cerrada y efectiva e implícitamente transmitida mediante sus aislamientos bajos. En la medida en que los códigos integrados no cumplen su objetivo, el orden se vuelve problemático a nivel de la organización social y a nivel de la persona. En la medida en que los códigos integrados alcanzan su objetivo de socialización, entonces tenemos como resultado el encubrimiento profundo de la solidaridad mecánica. Esto constituye una paradoja fundamental que tiene que ser enfrentada y explorada.

\section{Cambio del código educativo}

Hemos tratado de hacer explícitas las relaciones entre los códigos educativos, la estructura de poder y los principios de control. Los intentos por cambiar o modificar los códigos educativos se encuentran con resistencia en diferentes niveles independientemente del mérito educativo intrínseco a un código particular. Discutiremos brevemente a continuación algunas de las razones para el movimiento hacia la institucionalización de los códigos integrados con clasificación y enmarcación débiles

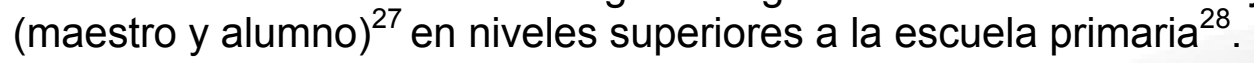

\footnotetext{
${ }^{27}$ En el artículo, hemos sugerido que los códigos integrados descansan sobre una ideología explícita cerrada. Se podría deducir que este código presentaría una mejor oportunidad de institucionalización exitosa en sociedades donde (1) existen restricciones fuertes y efectivas sobre el desarrollo de diferentes ideologías y (2) donde el sistema educativo es la principal agencia de socialización política. Además, los procedimientos de mantenimiento de la delimitación débil de los códigos integrados incrementaría la penetración de la socialización en la medida en que una gran parte del yo se hace pública mediante la relajación del enmarcamiento y la delimitación haría más visibles las infracciones o desviaciones. Por otra parte, los códigos integrados conllevan la potencialidad para el cambio de las estructuras de poder y los principios de control. Asumiríamos por lo tanto que en tales sociedades los códigos integrados poseerían una clasificación débil pero los marcos para el maestro y el alumno serían fuertes.

${ }^{28}$ Es importante anotar que en Inglaterra, es sólo en la escuela pre-escolar donde ha habido una introducción relativamente amplia de esta forma de código integrado. Esto plantea la pregunta de cómo este nivel de educación (del sistema educativo) fue abierto a tal cambio. Históricamente la escuela primaria desarrolló distintos conceptos de niño y de adolescente y distintas direcciones para tales estados. Dada la relativa autonomía sobre la transmisión del conocimiento que caracteriza al sistema educativo británico, fue inicialmente posible, en principio, realizar tal cambio. A pesar de que sólo un techo puede separar el departamento infantil del departamento de los adolescentes, se pueden desarrollar dos códigos educativos muy distintos y a menudo incompatibles. Podemos mirar esta condición como necesaria pero no suficiente para el surgimiento de los códigos integrados en la escuela primaria. También se dio el caso, hasta hace poco, de que la función de selección comenzaba en el departamento de los jóvenes porque este departamento era la puerta de salida para las "grammar schools". Esto dejó la escuela infantil relativamente libre de control por niveles más altos, (superiores). La forma de integración en la escuela infantil, hasta hace poco, estaba basada en el maestro y por lo tanto no presentaba los problemas que surgen de la integración basada en los maestros. Finalmente, los maestros de la escuela infantil no son socializados dentro de identidades educativas fuertes. De esta manera, el sistema educativo inglés, hasta hace poco, tenía dos puntos potenciales de apertura - el período entre las edades de cinco y siete años, antes de que la selección comenzara, y el período posterior a los dieciocho años cuando la selección virtualmente se completaba. El control más importante sobre la estructuración del conocimiento en el nivel secundario es la estructuración del conocimiento en el nivel terciario, específicamente en la universidad. Sólo si hay un amplio cambio en la estructuración del conocimiento en este nivel puede haber efectivamente cambio en el código de los niveles inferiores; a pesar de que en cualquier escuela puede existir una variedad de códigos del conocimiento.
} 
1) La creciente diferenciación del conocimiento en los niveles más altos del conocimiento, junto a la integración de áreas previamente separadas, pueden fundamentar los requisitos para la creación de una forma de socialización apropiada a estos cambios en la estructura del conocimiento.

2) Los cambios en la división del trabajo están creando un concepto diferente de habilidad. La obsolescencia de todas las variedades de habilidades, reduce el significado de operaciones ligadas al contexto y aumenta el significado de principios generales a partir de los cuales una gran cantidad de operaciones diversas pueden ser derivadas. En términos escuetos, podría decirse que el siglo XIX requirió un hombre sumiso e inflexible, mientras que el siglo XX (particularmente en su última etapa) requiere la conformación de un hombre flexible.

3) La estructura social menos rígida del código integrado hace de éste un código potencial para el desarrollo de una igualdad educativa.

4) En las sociedades industriales avanzadas que permiten, dentro de ciertos límites, un número de creencias e ideologías legitimantes, es mayor el problema del control. Existe el problema de dar un sentido a lo débilmente coordinado y de cambiar los sistemas simbólicos, y existe el problema de la regulación interna de la persona. Los códigos integrados con su peso en la unidad subyacente del conocimiento, mediante su énfasis en la analogía y la síntesis podría verse como una respuesta al primer problema del "dar un sentido". El control inter-personal de los códigos integrados en oposición al control inter-posicional puede generar una forma de socialización intrusa y penetrante, bajo condiciones de ambigüedad en el sistema de creencias y del orden moral.

Si estas razones funcionan, podríamos considerar el movimiento hacia los códigos integrados, a partir de una fuente tecnológica. Sin embargo, es posible que exista otra fuente más profunda en el intento por abandonar los códigos agregados. Se puede sugerir que el movimiento de los códigos agregados hacia los integrados simboliza que hay una crisis en las clasificaciones y marcos básicos de la sociedad, y por lo tanto una crisis en su estructura de poder y principios de control. Desde este punto de vista, el movimiento hacia los códigos integrados representa un intento por des-clasificar, y de esta manera alterar, las estructuras de poder y los Principios de control; al hacer esto también se intenta descongelar la estructuración del conocimiento y cambiar las delimitaciones de la conciencia. Desde este punto de vista, los códigos integrados son síntomas de una crisis moral en vez de ser el estado terminal de un sistema educativo.

\section{Conclusión}

En este artículo hemos tratado de explorar el concepto de delimitación en tal forma que es posible ver tanto los componentes de poder como los de control. El análisis se centró directamente sobre la estructuración del conocimiento educativo que se transmite.

Aunque el concepto de "clasificación" parece operar en una sola dimensión, i.e. diferencias en los grados de aislamiento entre contenidos (materias/cursos, etc.) este apunta explícitamente hacia los componentes poder y control. En la misma forma, el concepto enmarcación parece operar en una sola dimensión; lo que puede o no puede ser enseñado en la relación pedagógica. Sin embargo, la exploración de este concepto apunta también a los componentes de poder y control. Mediante la definición de los códigos educativos en términos de las relaciones entre clasificación y enmarcamiento, estos dos componentes se construyen en el análisis de todos los niveles. De esta manera se 
hace posible derivar en un marco de trabajo una tipología de códigos educativos, mostrar las inter-relaciones entre las propiedades organizativas y las propiedades del conocimiento, pasar del nivel de análisis macro al nivel de análisis micro, relacionar los patrones internos de las instituciones educativas con los antecedentes sociales externos de tales patrones, y considerar los problemas de la estabilidad y el cambio. Al mismo tiempo se espera que el análisis haga explícitos los presupuestos subyacentes (tácitos) de los diversos códigos educativos. El análisis ha intentado mostrar a un nivel teórico las relaciones entre un orden simbólico particular y la estructura de la experiencia. Consideramos que el análisis ofrece una aproximación que es susceptible de exploración por diversos métodos a nivel empírico. Debe quedar claro que la aplicación específica de los conceptos requiere confirmación empírica en cada momento del análisis. Hemos tratado de no complicar los argumentos con referencias porque en muchos casos las evidencias requeridas no existen en una forma que se relacione directamente con la cadena de inferencias y por lo tanto sólo ofrecerían un soporte falso. Tenemos, por ejemplo, poco conocimiento de primera mano, que pueda relacionarse directamente con los aspectos del enmarcamiento en la forma como se utiliza el concepto en este artículo. Igualmente no tenemos conocimiento de las formas de interacción cotidiana realizada por los diferentes tipos de códigos integrados.

Esperamos que la clase de problemas presentados en esta aproximación teórica estimulen a los sociólogos de la educación a explorar tanto teórica como empíricamente la estructura del conocimiento educativo, el cual se considera como el rasgo distintivo de este campo de investigación.

\section{REFERENCIAS}

BERNSTEIN, B. (1967), "Open schools, Open society?” New Society, 14 sept.

BERNSTEIN, B., Petes, R. and. ELVIN, L. (1966), "Ritual in education", Philosophical Transactions of the Royal Society of London, Series B, 251, No. 772.

DAVIES, D.I. (1970a), "The management of Knowledge: a critique of the use of typologies in educationalSociology", Sociology 4, No. 1.

- (1970b), "Knowledge, education asid power", papes presentad to the Britísh Sociologicsl Association Annual Conerence. Durham.

DOUGLAS, M. (1966), Purity and Danger, Routledge \& Kegan Paul.

—(1970), Natural Symbols, Barrio \& Rockliff, The Cresset Press.

DURKHEIM, E. (1947), Qn the División of Labour in Society, Chicago: Free Press.

- (1956) Education and Sociology, Chicago: Free Presa. (Especially chs. 2 and. 3.).

—And MAUSS, M. (1963), Primitiva Classification (translated by Needham. R.X Cohen \& West.

HOYLE, E. (1969), "How does the curriculum change? (1) A proposal for enquiries. (2) Systems and strategies", J. Curriculum Studies I, Nos. 2 sud. 3. 
JEFFERY, G.B. (1950), The Unity of Knowledge: Reflections on the Universities of Cambridge and London, Cambridge Umv. Press.

KEDDIE, N.G. (1970), "The social basis of classroom knowledge", MA Dissertation, Inst. of Educ. London Unive.

MUSGRAVE, F. (1968), "The contribution of sociology to the study of the curnculum", tu Changing the curriculum, ed., Kexr, J.F., Univ. of London Press.

YOUNG, M. (1970), "Currlcula as socially organizad knowledge" in Knowledge and Control, (ed.) Young, M. Collier-MacMillan. 